\title{
Markov Chain Confidence Intervals and Biases
}

\author{
Yu Hang Jiang ${ }^{1}$, Tong Liu ${ }^{1}$, Zhiya Lou ${ }^{1}$, Jeffrey S. Rosenthal ${ }^{1}$, Shanshan Shangguan ${ }^{1}$, Fei Wang ${ }^{1} \&$ Zixuan Wu ${ }^{1}$ \\ ${ }^{1}$ Department of Statistical Sciences, University of Toronto, Canada \\ Correspondence: Jeffrey S. Rosenthal, Department of Statistical Sciences, University of Toronto, Canada
}

Received: September 27, 2021 Accepted: December 14, 2021 Online Published: December 21, 2021

doi:10.5539/ijsp.v11n1p29

URL: https://doi.org/10.5539/ijsp.v11n1p29

\begin{abstract}
We derive explicit asymptotic confidence intervals for any Markov chain Monte Carlo (MCMC) algorithm with finite asymptotic variance, started at any initial state, without requiring a Central Limit Theorem nor reversibility nor geometric ergodicity nor any bias bound. We also derive explicit non-asymptotic confidence intervals assuming bounds on the bias or first moment, or alternatively that the chain starts in stationarity. We relate those non-asymptotic bounds to properties of MCMC bias, and show that polynomially ergodicity implies certain bias bounds. We also apply our results to several numerical examples. It is our hope that these results will provide simple and useful tools for estimating errors of MCMC algorithms when CLTs are not available.
\end{abstract}

\section{Introduction}

Markov chain Monte Carlo (MCMC) is a very powerful tool for estimating and sampling from complicated high-dimensional distributions (see e.g. (Brooks, Gelman, Jones, \& Meng, 2011) and the many references therein). MCMC algorithms help researchers in a wide spectrum of fields, ranging from Bayesian statistics to finance to computer science to physics.

One of the biggest challenges when implementing MCMC algorithms is to evaluate the error of the estimate, which is crucial for generating accurate results, and can also help when deciding how many iterations of the chain should be run. The majority of the existing results for quantifying MCMC accuracy rely heavily on the Markov chain Central Limit Theorem (CLT). However, this CLT is only known to be valid under specific conditions like geometric ergodicity or reversibility, which do not always hold and can be difficult to verify (see e.g. (Häggström \& Rosenthal, 2007; Ibragimov \& Linnik, 1971; Jones, 2004; Latuszyński, Miasojedow, \& Niemiro, 2013)). In the reversible case, (Kipnis \& Varadhan, 1986) established the existence of a CLT for all reversible Markov chains which have finite asymptotic estimator variance. However, without reversibility, CLTs are more challenging. Sufficient but difficult-to-verify conditions are provided in e.g. (Tóth, 1986), (Meyn \& Tweedie, 2012, Theorem 17.0.1), (Maxwell \& Woodroofe, 2000), and (Rudolf \& Sprungk, 2020, Theorem 2.2). And, (Häggström, 2005) shows that CLTs might not exist for non-reversible chains under conditions where CLTs would be guaranteed in the reversible case.

There have also been extensive investigations of confidence intervals in the steady-state literature. These techniques can be split into three subcategories, outlined in (Law \& Kelton, 1984). The most common approaches are replication and batch means. Replication runs the simulation numerous times, treating each run as an i.i.d. sample, while batch means divides one long simulation into multiple batches (see e.g. (Alexopoulos \& Seila, 1996)). Another approach is to model the autoregressive structures within the stochastic process and estimate parameters needed for the confidence interval from the model. Assuming the process is covariance stationary, (Fishman, 1971) derives a $p^{\text {th }}$ order autoregressive model as the variance estimator, while an alternative variance estimator is proposed by (Heidelberger \& Welch, 1981) using spectral analysis. Lastly, the regeneration cycles method, first introduced in (Crane \& Iglehart, 1975), identifies points during the simulation at which the process "restarts" probabilistically. Afterwards, the regeneration epochs are viewed as independent random variables in order to derive the confidence interval. However, all of these methods require assumptions or calculations which can make them difficult to implement in practice.

The recent paper (Rosenthal, 2017) derived a simple MCMC confidence interval which does not require a CLT, using only Chebychev's inequality. That result required certain assumptions about how the estimator bias and variance grow with the number of iterations $n$, in particular that the bias is $o(1 / \sqrt{n})$. This assumption seemed mild, since it is generally believed that the estimator bias will be $O(1 / n)$ and hence $o(1 / \sqrt{n})$ (see e.g. page 21 of (Geyer, 2011)). However, questions were raised ((Betancourt, 2020)) about how to verify this assumption, and indeed we show herein (Section 4) that it might not always hold.

This paper seeks to simplify and weaken the assumptions in (Rosenthal, 2017), to make MCMC confidence intervals without CLTs more widely applicable. In Section 2, we derive a conservative asymptotic Markov chain confidence interval (Theorem 1) assuming only a finite asymptotic estimator variance as in (Kipnis \& Varadhan, 1986), without requiring any bias assumption nor reversibility nor stationarity nor a CLT; at significance level $\alpha=0.05$ it is just 2.3 times 
as wide as the confidence interval that would follow from a CLT. In Section 3, we instead fix the number of iterations $n$, and obtain corresponding non-asymptotic confidence intervals without CLT under slightly stronger assumptions. In Section 4, we consider the question of when the MCMC bias is or is not $o(1 / \sqrt{n})$, and show that this property does not always hold but is ensured by a polynomial ergodicity condition. In Section 5, we complete the proof of Theorem 1 by extending to non-stationary chains. In Section 6, we present some numerical examples to illustrate our results, and we close in Section 7 with a brief summary.

\section{Asymptotic MCMC Confidence Intervals}

Let $\left\{X_{n}\right\}$ be a $\phi$-irreducible ergodic Markov chain on the state space $\mathcal{X}$, with stationary distribution $\pi(\cdot)$. Let $h: \mathcal{X} \rightarrow \mathbb{R}$ be a measurable function, let $\pi(h)=\int_{x \in X} h(x) \pi(x) d x$ be the (finite) expression we wish to estimate, and let $e_{n}:=\frac{1}{n} \sum_{i=1}^{n} h\left(X_{i}\right)$ be our estimate. At significance level $\alpha$, we wish to find a conservative $1-\alpha$ confidence interval, i.e. an interval which contains $\pi(h)$ with probability at least $1-\alpha$. Using only a variance bound, we show the following:

Theorem 1: If $\lim \sup _{n \rightarrow \infty} n \operatorname{Var}\left(e_{n}\right) \leq B^{2}$ for some $B>0$, then for any $0<\alpha<1$ and $\epsilon>0$, and $\pi$-a.e. initial state $X_{0}=x \in \mathcal{X}$, the interval

$$
I_{n}:=\left(e_{n}-(1+\epsilon) n^{-1 / 2} \alpha^{-1 / 2} B, e_{n}+(1+\epsilon) n^{-1 / 2} \alpha^{-1 / 2} B\right)
$$

is an asymptotic conservative $1-\alpha$ confidence interval for $\pi(h)$, i.e.

$$
\liminf _{n \rightarrow \infty} P\left[\pi(h) \in I_{n}\right] \geq 1-\alpha .
$$

Proof. First assume the chain starts in stationarity, so $E\left(e_{n}\right)=\pi(h)$ for all $n \in \mathbb{N}$. Then for any $a_{n}>0$, we have from Chebychev's inequality that

$$
P\left(\left|e_{n}-\pi(h)\right| \geq a_{n}\right)=P\left(\left|e_{n}-E\left(e_{n}\right)\right| \geq a_{n}\right) \leq \operatorname{Var}\left(e_{n}\right) / a_{n}^{2} .
$$

Therefore, setting $a_{n}=B / \sqrt{n \alpha}>0$ gives

$$
\begin{aligned}
\limsup _{n \rightarrow \infty} P\left(\left|e_{n}-\pi(h)\right| \geq a_{n}\right) & \leq \limsup _{n \rightarrow \infty}\left(\operatorname{Var}\left(e_{n}\right) / a_{n}^{2}\right) \\
& =\limsup _{n \rightarrow \infty}\left(\operatorname{Var}\left(e_{n}\right) \frac{n \alpha}{B^{2}}\right) \\
& \leq \limsup _{n \rightarrow \infty}\left(B^{2} \frac{\alpha}{B^{2}}\right)=\alpha .
\end{aligned}
$$

Then, taking complements gives

$$
\liminf _{n \rightarrow \infty} P\left(\left|e_{n}-\pi(h)\right|<n^{-1 / 2} \alpha^{-1 / 2} B\right)=\liminf _{n \rightarrow \infty} P\left(\left|e_{n}-\pi(h)\right|<a_{n}\right) \geq 1-\alpha .
$$

This proves the result (with $\epsilon=0$ ) assuming the chain starts in stationarity.

Finally, applying Theorem 5 from Section 5 below, with $\epsilon>0$ and $r=1 / 2$ and $C=\epsilon \alpha^{-1 / 2} B$, we obtain the result for $\pi$-a.e. $X_{0}=x \in \mathcal{X}$.

Theorem 1 says that any Markov chain satisfying $\lim \sup _{n \rightarrow \infty} n \operatorname{Var}\left(e_{n}\right) \leq B^{2}$ for some $B>0$ immediately has a specified asymptotic confidence interval, without requiring any CLT. It does not require any bias bound, so it provides a partial response to (Betancourt, 2020). It does still require a variance bound. Asymptotic variance estimators can be obtained in many different ways, including repeated runs, integrated autocorrelation times, batch means, window estimators, regenerations, and more (see e.g. Section 3 of (Geyer, 1992)), but they often require challenging conditions to ensure consistency ((Glynn \& Whitt, 1972; Hobert, Jones, Presnell, \& Rosenthal, 2002; Jones, Haran, Caffo, \& Neath, 2006; Flegal \& Jones, 2010)). Alternatively, the variance bound can be consistently estimated directly from simulations by sampling $M$ independent copies of the chain and computing the sample variance of the resulting $e_{n}$ values (see the examples in Section 6 below).

For example, at the usual significance level $\alpha=0.05$, taking $\epsilon=0.001$, Theorem 1 yields the asymptotic $95 \%$ confidence interval

$$
\left(e_{n}-4.48 B / \sqrt{n}, e_{n}+4.48 B / \sqrt{n}\right) .
$$

By contrast, if we knew that a CLT held and that $\lim _{n \rightarrow \infty} n \operatorname{Var}\left(e_{n}\right)=B^{2}$, then we could derive the $95 \%$ confidence interval

$$
\left(e_{n}-1.96 B / \sqrt{n}, e_{n}+1.96 B / \sqrt{n}\right) \text {. }
$$


The width of the first confidence interval is 2.3 times the second, but it does not require reversibility, nor the actual convergence of $n \operatorname{Var}\left(e_{n}\right)$ as $n \rightarrow \infty$.

Remark. Our theorems provide conservative confidence intervals, which might be larger than necessary, and have coverage probabilities larger than $1-\alpha$, and lead to running more MCMC iterations than necessary. However, we do not consider this to be a major problem. The main challenge of MCMC is to obtain a final answer together with a guarantee that it is sufficiently accurate. (For example, (Jones \& Hobert, 2001) seek some number of iterations $n^{\prime}$ for which the chain is within 0.01 of stationarity, not necessarily the smallest $\operatorname{such} n^{\prime}$.) And, conservative confidence intervals do provide such guarantees. As long as the required iterations can still be run in a reasonable time, there is no major disadvantage to running MCMC for somewhat longer than is actually required.

\section{Non-asymptotic MCMC Confidence Intervals}

The confidence intervals from Theorem 1 are only valid asymptotically as $n \rightarrow \infty$. That limitation is quite common for most MCMC confidence intervals, since large $n$ is required for a CLT to hold. However, since we are not using any CLT in our analysis, it is possible to obtain a precise non-asymptotic interval, in terms of an upper bound on the bias, as follows.

Theorem 2: Suppose for some fixed $n \in \mathbb{N}$, the chain satisfies the variance bound $n \operatorname{Var}\left(e_{n}\right) \leq B^{2}$ for some $B>0$, and also the bias bound $\left|E\left(e_{n}\right)-\pi(h)\right| \leq C$ for some $C \geq 0$. Then for any significance level $\alpha \in(0,1)$, setting $\delta=\frac{C}{\frac{B}{\sqrt{n \alpha}}+C} \in[0,1)$ and $a_{n}=\frac{B}{\sqrt{n \alpha}(1-\delta)}$, the fixed- $n$ interval

$$
I_{n}:=\left(e_{n}-a_{n}, e_{n}+a_{n}\right)
$$

is a non-asymptotic conservative $1-\alpha$ confidence interval, i.e.

$$
P\left[\pi(h) \in I_{n}\right] \geq 1-\alpha, \quad n \in \mathbb{N} .
$$

Proof. We first compute that

$$
\frac{\delta}{1-\delta}=\frac{C}{\frac{B}{\sqrt{n \alpha}}+C} \mid \frac{\frac{B}{\sqrt{n \alpha}}}{\frac{B}{\sqrt{n \alpha}}+C}=\frac{C \sqrt{n \alpha}}{B},
$$

and hence

$$
\delta a_{n}=\frac{\delta}{1-\delta} \frac{B}{\sqrt{n} \alpha}=\frac{C \sqrt{n \alpha}}{B} \frac{B}{\sqrt{n \alpha}}=C .
$$

Thus $\left|E\left(e_{n}-\pi(h)\right)\right| \leq C=\delta a_{n}$, and hence $a_{n}-\left|E\left(e_{n}\right)-\pi(h)\right| \geq a_{n}-C=(1-\delta) a_{n}>0$. Therefore, using the triangle inequality and then Chebyshev's inequality, we have that

$$
\begin{aligned}
P\left(\left|e_{n}-\pi(h)\right| \geq a_{n} \mid\right) & =P\left(\left|e_{n}-E\left(e_{n}\right)+E\left(e_{n}\right)-\pi(h)\right| \geq a_{n}\right) \\
& \leq P\left(\left|e_{n}-E\left(e_{n}\right)\right|+\left|E\left(e_{n}\right)-\pi(h)\right| \geq a_{n}\right) \\
& =P\left(\left|e_{n}-E\left(e_{n}\right)\right| \geq a_{n}-\left|E\left(e_{n}\right)-\pi(h)\right|\right) \\
& \leq \operatorname{Var}\left(e_{n}\right) /\left(a_{n}-\left|E\left(e_{n}\right)-\pi(h)\right|\right)^{2} \\
& \leq \operatorname{Var}\left(e_{n}\right) /\left((1-\delta) a_{n}\right)^{2} \\
& =\operatorname{Var}\left(e_{n}\right)\left(\frac{n \alpha}{B^{2}}\right) \leq B^{2} \frac{\alpha}{B^{2}}=\alpha .
\end{aligned}
$$

Taking complements gives $P\left[\pi(h) \in I_{n}\right] \geq 1-\alpha$, as claimed.

If the chain is in stationarity, or at least reaches stationarity within $n$ iterations, then the bias is zero, and we obtain:

Corollary 1: Let $n \in \mathbb{N}$ be a fixed time such that the chain is in stationarity after $n$ steps. Then if $n \operatorname{Var}\left(e_{n}\right) \leq B^{2}$ for some $B>0$, then for any significance level $0<\alpha<1$, the interval

$$
I_{n}:=\left(e_{n}-n^{-1 / 2} \alpha^{-1 / 2} B, e_{n}+n^{-1 / 2} \alpha^{-1 / 2} B\right)
$$

is a non-asymptotic conservative $1-\alpha$ confidence interval, i.e.

$$
P\left[\pi(h) \in I_{n}\right] \geq 1-\alpha .
$$


Proof. By the stationarity assumption, $\left|E\left(e_{n}\right)-\pi(h)\right|=0$, so we can apply Theorem 2 with $C=0$. It follows that $\delta=0$ and $a_{n}=n^{-\frac{1}{2}} \alpha^{-1 / 2} B$. The result then follows immediately from Theorem 2 .

The assumptions for the non-asymptotic bound in Theorem 2 and Corollary 1 are stronger than for the asymptotic bound of Theorem 1, since they require a bound on the bias or for the chain to be at stationarity after $n$ iterations. However, we will see in the next section that we can sometimes utilize properties such as polynomial ergodicity to help us establish a bound on bias. Also, in practice, MCMC users often approximately verify stationarity through a plethora of convergence diagnostics such as plots and renewal theory and non-parametric tests; see e.g. (Mengersen, Robert, \& GuihenneucJouyaux, 1999) for a review.

Next, we present a result which does not assume stationarity, nor require a bound on the bias, nor require a bound on the variance. But as a trade-off, it assumes a bound on an absolute first moment, which might be harder to verify. It could still be useful if e.g. the first moment condition can be linked to another property that is easier to satisfy, which could be explored in future research.

Theorem 3: Suppose for some fixed $n \in \mathbb{N}$ we have $E\left(\left|e_{n}-\pi(h)\right|\right) \leq \gamma_{n}$ for some constant $\gamma_{n}>0$. Then for any significance level $\alpha \in(0,1)$, the interval

$$
I_{n}:=\left(e_{n}-\gamma_{n} \alpha^{-1}, e_{n}+\gamma_{n} \alpha^{-1}\right)
$$

is a non-asymptotic conservative $1-\alpha$ confidence interval, i.e.

$$
P\left[\pi(h) \in I_{n}\right] \geq 1-\alpha .
$$

Proof. Setting $a_{n}=\gamma_{n} / \alpha>0$, we have by Markov's inequality that

$$
\begin{aligned}
P\left(\left|e_{n}-\pi(h)\right| \geq a_{n}\right) & \leq E\left(\left|e_{n}-\pi(h)\right|\right) / a_{n} \\
& =E\left(\left|e_{n}-\pi(h)\right|\right) \frac{\alpha}{\gamma_{n}} \\
& \leq\left(\gamma_{n} \frac{\alpha}{\gamma_{n}}\right)=\alpha .
\end{aligned}
$$

Taking complements,

$$
\begin{aligned}
P\left[\pi(h) \in I_{n}\right] & =P\left(\left|e_{n}-\pi(h)\right| \leq \gamma_{n} \alpha^{-1}\right) \\
& =P\left(\left|e_{n}-\pi(h)\right| \leq a_{n}\right) \geq 1-\alpha .
\end{aligned}
$$

In particular, if the $\gamma_{n}$ converge monotonically to zero, then we obtain a confidence interval which shrinks to a point as $n$ approaches infinity.

\section{The Order of MCMC Bias}

Since we are estimating the quantity $\pi(h)=\int_{x \in \mathcal{X}} h(x) \pi(x) d x$ by the Markov chain estimator $e_{n}:=\frac{1}{n} \sum_{i=1}^{n} h\left(X_{i}\right)$, the bias after $n$ iterations is given by $\operatorname{Bias}\left(e_{n}\right):=E\left(e_{n}\right)-\pi(f)$. As previously mentioned, the results in (Rosenthal, 2017) assumed this bias was $o(1 / \sqrt{n})$ since it is generally believed to be $O(1 / n)$ (see e.g. p. 21 of (Geyer, 2011)). However, this is not always the case:

Example 1: Consider the Markov chain with state space $\mathcal{X}=\{0,1,2,3, \ldots\}$, and transition probabilities given by $p_{0,0}=1$, and for all $n \geq 1, p_{n, 0}=1-\frac{\sqrt{n}}{\sqrt{n+1}}$ and $p_{n, n+1}=\frac{\sqrt{n}}{\sqrt{n+1}}$. Then the chain is $\phi$-irreducible (and aperiodic) with $\pi(x)=\phi(x)=$ $\delta_{0}(x)$, i.e. $\pi(0)=1$ and $\pi(x)=0$ for all $x \neq 0$. Assume $X_{0}=1$. We then compute that, for $n=1,2,3, \ldots$,

$$
P\left[X_{n} \neq 0\right]=P\left[X_{n}=n+1\right]=\prod_{i=1}^{n} \frac{\sqrt{i}}{\sqrt{i+1}}=\frac{1}{\sqrt{n+1}} .
$$

Thus,

$$
\lim _{n \rightarrow \infty} P\left[X_{n} \neq 0\right]=\lim _{n \rightarrow \infty} \frac{1}{\sqrt{n+1}}=0 .
$$

So, the chain will converge to $\pi(\cdot)$ (from any initial distribution). 
Next, consider the function on $\mathcal{X}$ defined by $f(0)=0$, and $f(x)=1$ for $x \geq 1$. Then $\pi(f)=f(0)=0$. It follows that

$$
\begin{aligned}
\operatorname{Bias}\left(e_{n}\right) & =E\left(e_{n}\right)-\pi(f)=E\left(e_{n}\right)=\frac{1}{n} \sum_{j=1}^{n} E\left[h\left(X_{j}\right)\right] \\
& =\frac{1}{n} \sum_{j=1}^{n}\left[f(j+1) P\left(X_{j}=j+1\right)+f(0) P\left(X_{j}=0\right)\right] \\
& =\frac{1}{n} \sum_{j=1}^{n} \frac{1}{\sqrt{j+1}} \geq \frac{1}{n} \sum_{j=1}^{n} \frac{1}{\sqrt{n+1}}=\frac{1}{\sqrt{n+1}} .
\end{aligned}
$$

On the other hand,

$$
\begin{aligned}
\operatorname{Bias}\left(e_{n}\right) & =\frac{1}{n} \sum_{j=1}^{n} \frac{1}{\sqrt{j+1}} \leq \frac{1}{n} \int_{0}^{n} x^{-\frac{1}{2}} d x \\
& =\left.\frac{1}{n} 2 x^{\frac{1}{2}}\right|_{x=0} ^{x=n}=\frac{1}{n}(2 \sqrt{n})=\frac{2}{\sqrt{n}} .
\end{aligned}
$$

That is, $\frac{1}{\sqrt{n+1}} \leq \boldsymbol{B i a s}\left(e_{n}\right) \leq \frac{2}{\sqrt{n}}$. In particular, the bias is $O(1 / \sqrt{n})$, but is not $O(1 / n)$ nor even $o(1 / \sqrt{n})$.

Example 1 raises the question of what conditions guarantee the bias to be $o(1 / \sqrt{n})$. We shall derive such a result for a class of Markov chains that are polynomially ergodic, defined as follows (see e.g. (Jarner \& Tweedie, 2003), (Jones, 2004)):

Definition: Let $\left\{X_{n}\right\}$ be a Markov chain with stationary distribution $\pi(\cdot)$, and let $\|\cdot\|$ be total variation distance. Then the chain is polynomially ergodic if there exists a function $M: \mathcal{X} \rightarrow[0, \infty)$ such that:

$$
\left\|P^{n}(x, \cdot)-\pi(\cdot)\right\| \leq M(x) n^{-m}, \quad x \in \mathcal{X}, x \in \mathbb{N} ;
$$

here $m>0$ is the order of the polynomial ergodicity.

Theorem 4: Let $\left\{X_{n}\right\}$ be a polynomially ergodic Markov chain of order $m>\frac{1}{2}$, with stationary distribution $\pi(\cdot)$. Suppose for some $D \in[0, \infty)$ and $f: \mathcal{X} \rightarrow \mathbb{R}$, we have $|f(x)| \leq D$. Then for any fixed initial state $X_{0}=x$, the absolute bias $\left|\operatorname{Bias}\left(e_{n}\right)\right|$ is $o\left(n^{-1 / 2}\right)$ as $n \rightarrow \infty$.

Proof. Let $X_{0}=x$. We compute that

$$
\begin{aligned}
\left|\operatorname{Bias}\left(e_{n}\right)\right| & =\left|E\left(e_{n}\right)-\pi(f)\right| \\
& \leq \frac{1}{n} \sum_{i=1}^{n}\left|E\left[f\left(X_{i}\right)\right]-\pi(f)\right| \\
& \leq \frac{1}{n} \sum_{i=1}^{n} \sup _{g: X \rightarrow \mathbf{R},|g(x)| \leq D}\left|E\left[g\left(X_{i}\right)\right]-\pi(g)\right| \\
& \leq \frac{1}{n} \sum_{i=1}^{n} 2 D\left\|P^{i}(x, \cdot)-\pi(\cdot)\right\| \\
& \leq \frac{1}{n} \sum_{i=1}^{n} 2 D M(x) i^{-m} \\
& =\frac{2 D M(x)}{n} \sum_{i=1}^{n} i^{-m} .
\end{aligned}
$$


Case 1: $\frac{1}{2}<m<1$. Then

$$
\begin{aligned}
\left|\operatorname{Bias}\left(e_{n}\right)\right| & \leq \frac{2 D M(x)}{n} \sum_{i=1}^{n} i^{-m} \\
& \leq \frac{2 D M(x)}{n} \int_{0}^{n} x^{-m} d x \\
& =\frac{2 D M(x)}{n} \cdot \frac{1}{1-m}\left(n^{1-m}-0^{1-m}\right) \\
& =\frac{2 D M(x)}{n} \cdot \frac{1}{1-m}\left(n^{1-m}\right)
\end{aligned}
$$

Therefore

$$
n^{1 / 2}\left|\operatorname{Bias}\left(e_{n}\right)\right| \leq \frac{2 D M(x)}{1-m} n^{1 / 2-m},
$$

which $\rightarrow 0$ as $n \rightarrow \infty$ since $m>1 / 2$ and $1-m>0$ and $D, M(x)<\infty$.

Case 2: $m \geq 1$. Find some $\beta$ such that $1 / 2<\beta<1 \leq m$. Then since $\sum_{i=1}^{n} i^{-m} \leq \sum_{i=1}^{n} i^{-\beta}$, it follows as above that

$$
\lim _{n \rightarrow \infty} n^{1 / 2}\left|\operatorname{Bias}\left(e_{n}\right)\right| \leq \lim _{n \rightarrow \infty} \frac{2 D M(x)}{1-\beta} n^{1 / 2-\beta}=0 .
$$

Remark: This result says the bias is $o(1 / \sqrt{n})$ for any polynomially ergodic chain of order more than $1 / 2$. In the context of Theorem 2, this means that we can always find a constant $C$ such that $\left|E\left(e_{n}\right)-\pi(h)\right| \leq C=\delta a_{n}$, since $a_{n}$ is $O(1 / \sqrt{n})$. Furthermore, if we can calculate an explicit value for $M(x)$, then we can obtain a value for $C$. As a specific example, if a chain has polynomial order $3 / 4=: m$, with initial state $X_{0}=: x$ satisfying $M(x)=2$, and variance bound $n \operatorname{Var}\left(e_{n}\right) \leq$ $4=: B^{2}$, and functional bound $|f(x)| \leq 5=: D$, then after $n=100$ iterations we will have $\left|\operatorname{Bias}\left(e_{n}\right)\right| \leq \frac{2 D M(x)}{n} \frac{1}{1-m} n^{1-m}=$ $(20 / n)(4)\left(n^{1 / 4}\right) \doteq 2.53=: C$, so we can apply Theorem 2 at significance level $\alpha=0.05$ to find that $\delta=\frac{n_{2.53}-m}{(2 / \sqrt{5})+2.53} \doteq 0.74$ and $a_{n}=2 /(\sqrt{5}(1-0.74)) \doteq 3.44$, giving the $95 \%$ confidence interval $\left(e_{n}-3.44, e_{n}+3.44\right)$ after 100 iterations.

\section{Extending to Non-Stationary Chains}

Theorem 1 above was initially proved assuming the chain started in stationarity. However, in practice MCMC is hardly ever started in stationarity, so accuracy bounds without this assumption are much more useful. We now prove a general result which says that asymptotic confidence intervals from stationarity can always be enlarged slightly to become asymptotic confidence intervals from arbitrary initial states.

Theorem 5: Consider an ergodic Markov chain $\left\{X_{n}\right\}$ on a state space $\mathcal{X}$ with stationary distribution $\pi(\cdot)$, functional $h$, and usual estimator $e_{n}$. Suppose the sequence $\left(e_{n}+a_{n}, e_{n}+b_{n}\right)$ is an asymptotic conservative $1-\alpha$ confidence interval for $\pi(h)$ when started in stationarity, i.e.

$$
\liminf _{n \rightarrow \infty} P\left(a_{n}<\pi(h)-e_{n}<b_{n}\right) \geq 1-\alpha, \quad X_{0} \sim \pi(\cdot) .
$$

Then for any $c>0$ and $0<r<1$, and $\pi$-a.e. initial state $x \in \mathcal{X}$, the sequence $\left(e_{n}+a_{n}-c n^{-r}, e_{n}+b_{n}+c n^{-r}\right)$ is an asymptotic conservative $1-\alpha$ confidence interval for the chain when started from the initial state $X_{0}=x$, i.e.

$$
\liminf _{n \rightarrow \infty} P\left(a_{n}-c n^{-r}<\pi(h)-e_{n}<b_{n}+c n^{-r}\right) \geq 1-\alpha, \quad X_{0}=x
$$

Proof. By ergodicity, for $\pi$-a.e. $x \in \mathcal{X}$, we have $\lim _{n \rightarrow \infty}\left\|P^{n}(x, \cdot)-\pi(\cdot)\right\|=0$. Hence, for fixed $\epsilon>0$ and $x \in \mathcal{X}$, we can find $m \in \mathbb{N}$ such that $\left\|P^{m}(x, \cdot)-\pi(\cdot)\right\| \leq \epsilon$. Let $\left\{X_{n}\right\}$ be our original chain with $X_{0}=x$, and let $\left\{X_{n}^{\prime}\right\}$ be a second copy of the chain in stationarity, i.e. with $X_{0}^{\prime} \sim \pi(\cdot)$ and hence $X_{n}^{\prime} \sim \pi(\cdot)$ for all $n$. By Proposition $3(\mathrm{~g})$ of (Roberts \& Rosenthal, 2004), we can jointly define $X_{m}$ and $X_{m}^{\prime}$ so that $P\left(X_{m}=X_{m}^{\prime}\right) \geq 1-\epsilon$, and we can then continue $\left\{X_{n}\right\}$ and $\left\{X_{n}^{\prime}\right\}$ together after time $m$ to get $P(H) \geq 1-\epsilon$ where

$$
H=\left\{X_{n}=X_{n}^{\prime} \text { for all } n \geq m\right\} .
$$

Now, let $e_{n}=\frac{1}{n} \sum_{i=1}^{n} h\left(X_{i}\right)$, and $e_{n}^{\prime}=\frac{1}{n} \sum_{i=1}^{n} h\left(X_{i}^{\prime}\right)$ be the estimators from the two chains, so by assumption we have

$$
\liminf _{n \rightarrow \infty} P\left(a_{n}<\pi(h)-e_{n}^{\prime}<b_{n}\right) \geq 1-\alpha .
$$


Then on the event $H$, for any $n \geq m$ we have

$$
\left|\left(\pi(h)-e_{n}\right)-\left(\pi(h)-e_{n}^{\prime}\right)\right|=\frac{1}{n}\left|\sum_{i=1}^{m}\left(h\left(X_{i}^{\prime}\right)-h\left(X_{i}\right)\right)\right|=: \frac{1}{n}|Z|,
$$

where $Z=\left|\sum_{i=1}^{m}\left(h\left(X_{i}^{\prime}\right)-h\left(X_{i}\right)\right)\right|$. Hence, if $H$ holds and $a_{n}<\pi(h)-e_{n}^{\prime}<b_{n}$ and $\frac{1}{n}|Z| \leq c n^{-r}$, then $a_{n}-c n^{-r}<\pi(h)-e_{n}<$ $b_{n}+c n^{-r}$. Furthermore, $Z$ is a fixed finite random variable, so there is $A<\infty$ with $P(|Z|>A) \leq \epsilon$. It follows that for $n \geq(A / c)^{1 /(1-r)}$, we have

$$
P\left(\frac{1}{n}|Z|>c n^{-r}\right)=P\left(|Z|>c n^{1-r}\right) \leq P(|Z|>A) \leq \epsilon .
$$

We conclude that for all $n \geq \max \left[m,(A / c)^{1 /(1-r)}\right]$,

$$
\begin{aligned}
& P\left(\left\{a_{n}-c n^{-r}<\pi(h)-e_{n}<b_{n}+c n^{-r}\right\}^{C}\right) \\
& \leq P\left(\left\{a_{n}<\pi(h)-e_{n}^{\prime}<b_{n}\right\}^{C}\right)+P\left(H^{C}\right)+P(|Z|>A) \\
& \leq P\left(\left\{a_{n}<\pi(h)-e_{n}^{\prime}<b_{n}\right\}^{C}\right)+\epsilon+\epsilon,
\end{aligned}
$$

i.e.

$$
P\left(a_{n}-c n^{-r}<\pi(h)-e_{n}<b_{n}+c n^{-r}\right) \geq P\left(a_{n}<\pi(h)-e_{n}^{\prime}<b_{n}\right)-2 \epsilon .
$$

Then, taking lim inf gives

$$
\liminf _{n \rightarrow \infty} P\left(a_{n}-c n^{-r}<\pi(h)-e_{n}<b_{n}+c n^{-r}\right) \geq \alpha-2 \epsilon .
$$

Since this is true for any $\epsilon>0$, we must actually have

$$
\liminf _{n \rightarrow \infty} P\left(a_{n}-c n^{-r}<\pi(h)-e_{n}<b_{n}+c n^{-r}\right) \geq \alpha,
$$

giving the result.

\section{Numerical Examples}

In this section, we apply Theorem 1 to various non-reversible examples, to obtain confidence intervals directly without the need to establish a CLT nor any convergence rates.

\subsection{A Cyclical Non-Reversible Chain}

Define a Markov chain on the state space $\mathcal{X}=\mathbb{N}$, as follows. Fix $0<r<1$. For all $x>1$, let $p_{1, x}=r^{x-2}(1-r)$, and $p_{x, x-1}=1$, with $p_{x, y}=0$ otherwise. It is easily verified that this chain has stationary distribution given by:

$$
\begin{aligned}
& \pi(1)=\pi(2)=\frac{1-r}{r^{2}-2 r+2}, \\
& \pi(x)=\left(\frac{1-r}{r^{2}-2 r+2}\right) r^{x-1}, \quad x>2 .
\end{aligned}
$$

Furthermore, this chain is irreducible and aperiodic. Hence, the chain will converge asymptotically to $\pi$, and furthermore $e_{n}$ will converge to $\pi(h)$ whenever $\pi|h|<\infty$. However, it is not trivial to establish a confidence interval for $\pi(h)$ in terms of $e_{n}$ by means of a CLT, since this chain is clearly non-reversible, and establishing a condition like geometric ergodicity would require careful drift function arguments. Instead, we use our method. Take $X_{0}=1, r=0.75$, and $h(x)=x^{0.5}$. For different numbers of iterations $n$, we run $M=100$ replications to estimate $n \operatorname{Var}\left(e_{n}\right)$ and $e_{n}$. Our results are as follows:

\begin{tabular}{|c|c|c|}
\hline$n$ & $n \widehat{\operatorname{Var}}\left(e_{n}\right)$ & $\widehat{e_{n}}$ \\
\hline 1,000 & 2.840 & 1.91171 \\
2,000 & 3.413 & 1.90454 \\
5,000 & 4.054 & 1.90518 \\
10,000 & 2.557 & 1.90171 \\
20,000 & 3.299 & 1.90384 \\
50,000 & 2.611 & 1.90501 \\
100,000 & 3.514 & 1.90434 \\
200,000 & 4.323 & 1.90414 \\
500,000 & 3.040 & 1.90433 \\
$1,000,000$ & 3.596 & 1.90416 \\
\hline
\end{tabular}


These replications indicate that $n \operatorname{Var}\left(e_{n}\right) \leq 5$ for all $n$. Hence, we can take $B=\sqrt{5}$. Then, setting $\alpha=0.05$ and $\epsilon=0.001$, Theorem 1 gives an approximate conservative $95 \%$ confidence interval for $\pi(h)$ equal to:

$$
I \equiv I_{1,000,000}=(1.894,1.914) .
$$

This is quite a small interval, of width 0.02 , which provides good confidence about $\pi(h)$, without needing to establish any CLT or difficult ergodicity property.

\subsection{A Diffusive Non-Reversible Chain}

Consider the Markov chain with state space $\mathcal{X}=\{0,1,2,3, \ldots\}$, and transition probability given by $p_{0,0}=0.99, p_{0,1}=$ 0.01 , and for all $x \geq 1, p_{x, x+1}=\left(\frac{x}{x+1}\right)^{2}$ and $p_{x, 0}=1-\left(\frac{x}{x+1}\right)^{2}$. This chain is easily computed to have stationary distribution:

$$
\pi(0)=\frac{1}{1+\frac{0.01 \pi^{2}}{6}}, \quad \text { and } \quad \pi(x)=\frac{0.01}{x^{2}} \pi(0) \text { for } x \geq 1 .
$$

This chain is again irreducible and aperiodic, so again $e_{n}$ will converge to $\pi(h)$ whenever $\pi|h|<\infty$. However, this chain is again clearly non-reversible, and it is again non-trivial to establish a CLT to obtain a confidence interval for $\pi(h)$ in terms of $e_{n}$. Instead, we again use our method. Let

$$
h(x)= \begin{cases}0, & x=0 \\ x^{-1}, & x \geq 1\end{cases}
$$

and again take $X_{0}=1$. For different numbers of iterations $n$, we run $M=1,000$ replications to estimate $e_{n}$ and its variance. The results are as follows:

\begin{tabular}{|c|c|c|}
\hline$n$ & $n \widehat{\operatorname{Var}}\left(e_{n}\right)$ & $\widehat{e_{n}}$ \\
\hline 100 & 0.0181 & 0.01400 \\
200 & 0.0163 & 0.01264 \\
500 & 0.0148 & 0.01224 \\
1,000 & 0.0156 & 0.01196 \\
2,000 & 0.0151 & 0.01188 \\
5,000 & 0.0151 & 0.01195 \\
10,000 & 0.0142 & 0.01185 \\
20,000 & 0.0153 & 0.01184 \\
50,000 & 0.0154 & 0.01186 \\
100,000 & 0.0141 & 0.01182 \\
\hline
\end{tabular}

These simulations indicate that $n \operatorname{Var}\left(e_{n}\right) \leq 0.02$ for all $n$, so we can take $B=\sqrt{0.02}$. Then, setting $\alpha=0.05$ and $\epsilon=0.001$, Theorem 1 gives an approximate conservative $95 \%$ confidence interval for $\pi(h)$ equal to:

$$
I \equiv I_{100,000}=(0.0098,0.0138)
$$

This is again quite a small interval, of width 0.004 , again providing good confidence about $\pi(h)$, without needing to establish any CLT or difficult ergodicity property.

\subsection{A Polynomial-Tailed Non-Reversible Chain}

Define a Markov chain on the state space $\mathcal{X}=\mathbb{N}$, as follows. Fix $s>2$. For all $x>1$, let $p_{1, x}=x^{-s}$, and $p_{x, x-1}=1$, with $p_{x, y}=0$ otherwise. Let $\zeta(s)=\sum_{i=1}^{\infty} i^{-s}$ denote the Riemann zeta function of $s$. It follows by induction that its stationary distribution $\pi$ satisfies that

$$
\pi(x)=\left(\sum_{i=x}^{\infty} i^{-s}\right) \pi(1), \quad x>1 .
$$

Then, normalising the measure, we conclude that

$$
\begin{aligned}
& \pi(1)=\frac{1}{\zeta(s-1)-\zeta(s)+1} \\
& \pi(x)=\frac{\sum_{i=x}^{\infty} i^{-s}}{\zeta(s)-\zeta(s-1)+1}, \quad x>1 .
\end{aligned}
$$


This chain is again non-reversible, and is again irreducible and aperiodic so $e_{n}$ will converge to $\pi(h)$ whenever $\pi(|h|)<\infty$. The polynomial tails of $p_{x, 1}$ and $\pi$ make it difficult to directly establish a CLT, so we again proceed through simulation. Let $X_{0}=1, s=5$, and $h(x)=x$. For different numbers of iterations $n$, we run $M=1000$ replications of the chain to estimate $n \operatorname{Var}\left(e_{n}\right)$ and $e_{n}$.

\begin{tabular}{|c|c|c|}
\hline$n$ & $n \widehat{\operatorname{Var}}\left(e_{n}\right)$ & $\widehat{e_{n}}$ \\
\hline 100 & 0.552 & 1.61442 \\
200 & 0.807 & 1.61220 \\
500 & 1.013 & 1.61519 \\
1,000 & 0.946 & 1.61516 \\
2,000 & 0.874 & 1.61484 \\
5,000 & 0.883 & 1.61500 \\
10,000 & 0.774 & 1.61444 \\
20,000 & 0.855 & 1.61436 \\
50,000 & 0.872 & 1.61441 \\
100,000 & 0.892 & 1.61458 \\
\hline
\end{tabular}

The simulations indicate that $n \operatorname{Var}\left(e_{n}\right) \leq 1.5$ for all $n$. Therefore, we can take $B=\sqrt{1.5}$. Then, setting $\alpha=0.05$ and $\epsilon=0.001$, Theorem 1 gives an approximate conservative $95 \%$ confidence interval for $\pi(h)$ equal to:

$$
I \equiv I_{100,000}=(1.597,1.632)
$$

which is again quite a small interval, providing good confidence about $\pi(h)$.

\subsection{A Discretised Irreversible Langevin Diffusion}

Finally, we examine a discrete version of the Irreversible Langevin sampler for $X_{t} \in \mathbb{R}^{2}$ introduced in (Rey-Bellet \& Spiliopoulos, 2015). Recall first the standard (reversible) Langevin diffusion, defined by:

$$
d X_{t}=-\nabla U\left(X_{t}\right) d t+\sqrt{2 D} d W_{t},
$$

where $U: \mathbb{R}^{2} \rightarrow \mathbb{R}$ is a $C^{1}$ function, $D>0$ is a constant, and $W_{t}$ is two-dimensional Brownian motion. This process converges to the stationary distribution having density

$$
\pi(x, y)=\frac{e^{-U(x, y) / D}}{\int_{\mathbb{R}^{2}} e^{-U(x, y) / D} d x d y} .
$$

(Rey-Bellet \& Spiliopoulos, 2015) show that the non-reversible family of diffusions

$$
d X_{t}=\left[-\nabla U\left(X_{t}\right)+C\left(X_{t}\right)\right] d t+\sqrt{2 D} d W_{t}
$$

also converges to this same stationary distribution $\pi$ provided that the $C^{1}$ vector field $C(x, y) \operatorname{satisfies} \operatorname{div}\left(C e^{-2 U}\right)=0$, and this condition is guaranteed if $C(x, y)=J \nabla U(x, y)$ for some antisymmetric matrix $J$. This new process is no longer reversible, but they argue that it will sometimes converge faster.

We now consider using this non-reversible Langevin diffusion to estimate $\pi(h)$ for some function $h: \mathbb{R}^{2} \rightarrow \mathbb{R}$. We run a discrete-time version of this continuous-time Irreversible Langevin process, replacing each $d t$ by 0.001 , and each $d W_{t}$ by an independent $N(0, d t)$ draw. We take $X_{0}=(0,0), U(x, y)=\left(x^{2}-2\right)^{2}+\frac{1}{4} y^{2}, D=0.1, C(x, y)=\left[\begin{array}{cc}0 & 10 \\ -10 & 0\end{array}\right] \nabla U(x, y)$, and $h(x, y)=16 x^{2}+9 y^{2}$. We start each run at $t=0$ and run until $t=T$ for $T \in\{10,20, \ldots, 100\}$. Due to the complexity of the model, we discard the results for $0 \leq t \leq 5$ as burn-in to minimize the effect of bias. Lastly, we define $n:=(T-5) / d t$, and run $M=100$ replications for each $T$ to estimate $e_{n}$ and $n \operatorname{Var}\left(e_{n}\right)$. Our results are as follows: 


\begin{tabular}{|c|c|c|c|}
\hline$T$ & $n$ & $n \widehat{\operatorname{Var}}\left(e_{n}\right)$ & $\widehat{e_{n}}$ \\
\hline 10 & 5,000 & 0.04666 & 32.00178 \\
20 & 15,000 & 0.02192 & 32.00156 \\
30 & 25,000 & 0.02074 & 32.00165 \\
40 & 35,000 & 0.02345 & 32.00173 \\
50 & 45,000 & 0.01570 & 32.00165 \\
60 & 55,000 & 0.01450 & 32.00164 \\
70 & 65,000 & 0.01668 & 32.00154 \\
80 & 75,000 & 0.01769 & 32.00168 \\
90 & 85,000 & 0.01612 & 32.00158 \\
100 & 95,000 & 0.01721 & 32.00172 \\
\hline
\end{tabular}

These simulations indicate that $n \operatorname{Var}\left(e_{n}\right) \leq 0.05$ for all $n$. Therefore, we can take $B=\sqrt{0.05}$. Then, setting $\alpha=0.05$ and $\epsilon=0.001$, Theorem 1 gives an approximate conservative $95 \%$ confidence interval for $\pi(h)$ equal to:

$$
I \equiv I_{95,000}=(31.998,32.005)
$$

an extremely narrow interval (width 0.007 ) which gives good confidence about the value of $\pi(h)$, again without proving any CLT or any challenging ergodicity property.

\section{Summary}

In this paper, we have derived explicit asymptotic confidence intervals for any MCMC algorithm with finite asymptotic variance, started at any initial state, without requiring a Central Limit Theorem nor reversibility nor any bias bound. We have also derived explicit non-asymptotic confidence intervals assuming bounds on the bias or first moment, or alternatively that the chain starts in stationarity. We have related those non-asymptotic bounds to properties of MCMC bias, and shown that polynomially ergodicity implies appropriate bias bounds. Finally, we have applied our results to several numerical examples. It is our hope that these results will provide simple and useful tools for estimating errors of MCMC algorithms when CLTs are not easily available.

\section{Acknowledgements}

This research was partially supported by NSERC of Canada discovery grant RGPIN-2019-04142.

\section{References}

Alexopoulos, C., \& Seila, A. F. (1996). Implementing the batch means method in simulation experiments. In Proceedings of the 28th conference on winter simulation (p. 214221). USA: IEEE Computer Society.

Betancourt, M. (2020). Reply tweet of May 25, 2020. Twitter.

Brooks, S., Gelman, A., Jones, G., \& Meng, X. L. (2011). Handbook of Markov chain Monte Carlo. CRC press.

Crane, M. A., \& Iglehart, D. L. (1975). Simulating stable stochastic systems: Iii. regenerative processes and discrete-event simulations. Operations Research, 23(1), 33-45.

Fishman, G. S. (1971). Estimating sample size in computing simulation experiments. Management Science, 18(1), 21-38.

Flegal, J. M., \& Jones, G. L. (2010). Batch means and spectral variance estimators in Markov chain Monte Carlo. The Annals of Statistics, 38(2), 1034-1070.

Geyer, C. (1992). Practical Markov chain Monte Carlo. Statistical Science, 7, 473-483.

Geyer, C. (2011). Introduction to Markov chain Monte Carlo. Handbook of Markov chain Monte Carlo, 20116022, 45.

Glynn, P. W., \& Whitt, W. (1972). The asymptotic validity of sequential stopping rules for stochastic simulations. The Annals of Applied Probability, 2(1), 180-198.

Häggström, O. (2005, May 01). On the Central Limit Theorem for geometrically ergodic Markov chains. Probability Theory and Related Fields, 132(1), 74-82.

Häggström, O., \& Rosenthal, J. S. (2007). On variance conditions for Markov chain CLTs. Electronic Communications in Probability, 12, 454-464.

Heidelberger, P., \& Welch, P. D. (1981, April). A spectral method for confidence interval generation and run length control in simulations. Commun. ACM, 24(4), 233245.

Hobert, J., Jones, G., Presnell, B., \& Rosenthal, J. (2002). On the applicability of regenerative simulation in Markov chain Monte Carlo. Biometrika, 89, 731-743.

Ibragimov, I. A., \& Linnik, Y. V. (1971). Independent and stationary sequences of random variables,(1971). WaltersNoordhoff, Gröningen. 
Jarner, S. F., \& Tweedie, R. L. (2003, 08). Necessary conditions for geometric and polynomial ergodicity of randomwalk-type. Bernoulli, 9(4), 559-578.

Jones, G. L. (2004). On the Markov chain Central Limit Theorem. Probability Surveys, 1, 299-320.

Jones, G. L., Haran, M., Caffo, B., \& Neath, R. (2006). Fixed-width output analysis for Markov chain Monte Carlo. Journal of the American Statistical Association, 101(476), 1537-1547.

Jones, G. L., \& Hobert, J. P. (2001). Honest exploration of intractable probability distributions via Markov chain Monte Carlo. Statistical Science, 312-334.

Kipnis, C., \& Varadhan, S. R. S. (1986). Central limit theorem for additive functionals of reversible Markov processes and applications to simple exclusions. Communications in Mathematical Physics, 104(1), 1-19.

Latuszyński, K., Miasojedow, B., \& Niemiro, W. (2013). Nonasymptotic bounds on the estimation error of MCMC algorithms. Bernoulli, 19(5A), 2033-2066.

Law, A. M., \& Kelton, W. D. (1984). Confidence intervals for steady-state simulations: I. a survey of fixed sample size procedures. Operations Research, 32(6), 1221-1239.

Maxwell, M., \& Woodroofe, M. (2000). Central Limit Theorems for additive functionals of Markov chains. Ann. Prob., $28(2), 713-724$.

Mengersen, K. L., Robert, C. P., \& Guihenneuc-Jouyaux, C. (1999). MCMC convergence diagnostics: a review. Bayesian Statistics, 6, 415-440.

Meyn, S., \& Tweedie, R. (2012). Markov chains and stochastic stability. Springer Science \& Business Media.

Rey-Bellet, L., \& Spiliopoulos, K. (2015, 5). Irreversible Langevin samplers and variance reduction: a large deviations approach. Nonlinearity (Print), 28(7).

Roberts, G. O., \& Rosenthal, J. S. (2004). General state space Markov chains and MCMC algorithms. Probability Surveys, $1,20-71$.

Rosenthal, J. S. (2017). Simple confidence intervals for MCMC without CLTs. Electron. J. Statist., 11(1), 211-214.

Rudolf, D., \& Sprungk, B. (2020). On a Metropolis-Hastings importance sampling estimator. Elec. J. Stat., 14(2), $857-889$.

Tóth, B. (1986, Dec 01). Persistent random walks in random environment. Probability Theory and Related Fields, 71(4), 615-625.

\section{Copyrights}

Copyright for this article is retained by the author(s), with first publication rights granted to the journal.

This is an open-access article distributed under the terms and conditions of the Creative Commons Attribution license (http://creativecommons.org/licenses/by/4.0/). 